\title{
Conscientização sobre hábitos relacionados ao sono no interior do estado de São Paulo
}

\author{
Conscientization of the habits related to sleep inside \\ the state of São Paulo \\ Conscientización de los hábitos relacionados con el sueño en \\ el interior del estado de São Paulo \\ Camila de Castro CORRÊA ${ }^{1}$ \\ Leticia Dominguez CAMPOS ${ }^{2}$ \\ Silke Anna Theresa WEBER \\ ${ }^{1}$ Doutoranda pelo Programa Bases Gerais da Cirurgia, Faculdade de Medicina de Botucatu, Univ Estadual Paulista UNESP \\ 18618-687, Botucatu-SP, Brasil \\ ${ }^{2}$ Professora Assistente, Curso de Fisioterapia, Faculdade Marechal Rondon FMR - 18650-00, São Manuel-SP, Brasil \\ ${ }^{3}$ Professora Adjunto, Departamento de Oftalmologia e Otorrinolaringologia, Faculdade de Medicina de Botucatu \\ Univ Estadual Paulista - UNESP, 18618-687 Botucatu - SP, Brasil
}

\begin{abstract}
Resumo
Introdução: Na semana do dia 15 de março, são realizadas ações pelo mundo para a conscientização sobre o sono. Para tal missão, a Associação Brasileira do Sono conta com a coordenação de um membro associado por cidade, responsável por organizar atividades na sua região. Objetivo: descrever as ações de promoção de saúde desenvolvidas nas cidades do interior paulista na semana do sono do ano de 2017. Métodos: Foram realizadas parcerias com universidades, hospitais, empresas e estabelecimentos comerciais para a realização de palestras com conteúdos gerais de higiene do sono, a influência das tecnologias de comunicação na qualidade do sono e conteúdos específicos do sono em trabalhadores de turno. Resultados: As palestras aconteceram nos seguintes locais: FEMSA Cocacola, Bauru/SP, Faculdade de Odontologia de Bauru USP, Bauru/SP, Faculdade de Agudos FAAG, Agudos/SP, Universidade do Sagrado Coração USC, Bauru/SP, Faculdade Marechal Rondon, São Manuel/SP, Hospital das Clínicas - Faculdade de Medicina de Botucatu, Botucatu/SP, Hospital Estadual de Botucatu, Botucatu/SP, Açaí da Barra / Avenida Getúlio Vargas, Bauru/SP, Caminhada do $2^{\circ}$ movimento pro sono, Bauru/SP e Coca-cola Lençóis Paulista/SP. Dessa forma, as ações abrangeram desde crianças, adolescentes, universitários e idosos de cinco cidades do interior paulista. Foram abordadas 489 pessoas diretamente, além da disponibilização de cartilhas extras nesses mesmos ambientes em áreas de grande circulação de pessoas. Conclusão: As ações do interior paulista ocorreram de modo abrangente, atuando na população universitária até com grupos de idosos. Sugere-se que ações como esta sejam replicadas, abrangendo número ainda maior de participantes, em diferentes regiões.

Descritores: Sono; Promoção da Saúde; Exposições Educativas.
\end{abstract}

\section{Abstract}

Introduction: In the week of March 15, actions around the world for awareness about sleep are carried out. For this mission, the Brazilian Association of Sleep has the coordination of one associate member per city, responsible for organizing activities in its region. Objective: to describe the actions of health promotion developed in the cities of the state of São Paulo during the Sleep Awareness Week of the year 2017. Methods: Partnerships with universities, hospitals, companies and commercial establishments were held for the performance of lectures with general contents of sleep hygiene, the influence of communication technologies on sleep quality and specific sleep content in shift workers. Results: The lectures took place in the following locations: FEMSA CocaCola, Bauru / SP, Bauru Dental School, USP, Bauru/SP, Agudos Faculty, Agudos/SP, USC Sacred Heart University, Bauru/SP, Marechal Rondon College, São Manuel/SP, Clinics Hospital - Botucatu Medical School, Botucatu/SP, Botucatu State Hospital, Botucatu/SP, Açaí da Barra/Getulio Vargas Avenue, Bauru/SP, Walk of the 2nd movement to sleep, Bauru/SP and Coca-cola Lençóis Paulista/SP. In this way, the actions included children, adolescents, university students and elderly people from five cities of the interior of São Paulo. A total of 489 people were approached directly, besides the availability of extra booklets in these same environments in areas of great circulation of people. Conclusion: The actions of the interior of São Paulo occurred in a comprehensive way, acting in the university population even with groups of elderly people. It is suggested that actions such as this be replicated, involving an even greater number of participants, in different regions.

Descriptors: Sleep; Health Promotion; Health Fairs.

\section{Resumen}

Introducción: En la semana del 15 de marzo, se realizan acciones por el mundo para la concientización sobre el sueño. Para tal misión, la Asociación Brasileña del Sueño cuenta con la coordinación de un miembro asociado por ciudad, responsable de organizar actividades en su región. Objetivo: describir las acciones de promoción de salud desarrolladas en las ciudades del interior paulista en la semana del sueño del año 2017. Métodos: Se realizaron alianzas con universidades, hospitales, empresas y establecimientos comerciales para la realización de charlas con contenidos generales de higiene del sueño, la influencia de las tecnologías de comunicación en la calidad del sueño y contenidos específicos del sueño en trabajadores de turno. Resultados: Las conferencias se realizaron en los siguientes lugares: FEMSA Coca-cola, Bauru/SP, Facultad de Odontología de Bauru USP, Bauru/SP, Facultad de Agudos FAAG, Agudos/SP, Universidad del Sagrado Corazón USC, Bauru/SP, Facultad Marechal Rondon, São Manuel/SP, Hospital de Clínicas - Facultad de Medicina de Botucatu, Botucatu/SP, el Hospital Estatal de Botucatu, Botucatu/SP, Acai Bar, Bauru/SP, Caminata del $2^{\circ}$ movimiento del sueño, Bauru/SP y Coca-Cola, Lençóis Paulista/SP. De esta forma, las acciones abarcar desde niños, adolescentes, universitarios y ancianos de cinco ciudades del interior paulista. De esta forma, se abordaron 489 personas directamente, además de la disponibilidad de cartillas adicionales en esos mismos ambientes en áreas de gran circulación de personas. Conclusión: Las acciones del interior paulista ocurrieron de modo integral, actuando en la población universitaria hasta con grupos de ancianos. Se sugiere que acciones como ésta sean replicadas, abarcando el número aún mayor de participantes, en diferentes regiones.

Descriptores: Sueño; Promoción de la Salud; Exposiciones Educacionales en Salud.

\section{INTRODUÇÃO}

As alterações do sono afetam a qualidade de vida e a capacidade de execução das atividades de vida diária do indivíduo acometido ${ }^{1}$. O tema tem ganhado especial importância nos últimos anos, devido à alta prevalência de desordens do sono na população, bem como por suas sérias consequências à saúde ${ }^{2}$. A literatura mostra que as alterações do sono implicam em alterações fisiológicas expressivas para o desenvolvimento e estado geral do indivíduo, podendo haver, em consequência, comprometimento cognitivo, risco aumentado de acidentes de trânsito e de trabalho ${ }^{3-5}$, risco aumentado de desenvolver e/ou agravar doenças cardiovasculares e diabetes, estando associado a aumento da mortalidade ${ }^{6,7}$.

Apesar das amplas consequências das alterações do 
sono, frequentemente são desconhecidas pela população, ou mesmo pelos profissionais da saúde que não atuam diretamente na área. Desta forma, ações de promoção de saúde sobre o tema se fazem necessárias, não só para prevenção de alterações de sono, como também para melhorar o acesso ao diagnóstico dos distúrbios relacionados ao sono e a aderência aos tratamentos ${ }^{8,9}$.

Desde 2008, o "Dia Mundial do Sono" (World Sleep Day) é promovido pela World Association of Sleep Medicine (WASM). No Brasil, a "Semana do Sono" é organizada pela Associação Brasileira do Sono (ABS), Associação Brasileira de Medicina do Sono (ABMS) e Associação Brasileira de Odontologia do Sono (ABROS). Na semana do dia 15 de março, são realizadas ações pelo mundo para a conscientização de aspectos de prevenção de distúrbios do sono, além da procura precoce pelo tratamento quando necessário. Para tal missão, a ABS conta com a coordenação de um membro associado por cidade (interessado a participar), responsável por organizar atividades na sua região. Em 2017, o tema da campanha foi "Sonhos bons nutrem a vida" e, de acordo com divulgação da ABS, pelo menos 19 cidades brasileiras participaram das atividades, coordenadas por um membro associado ${ }^{10}$

O objetivo do presente estudo foi descrever as ações de promoção de saúde desenvolvidas nas cidades do interior do Estado de São Paulo durante a semana do sono do ano de 2017.

\section{MATERIAL E MÉTODO}

A coordenação das atividades da região de Bauru foi representada por uma fonoaudióloga, que realizou parceria com uma fisioterapeuta de São Manuel e uma médica otorrinolaringologista de Botucatu, especialista em Medicina do Sono, para o planejamento das ações da semana do sono, entre os dias 13 e 19 de março de 2017, nas cidades da região.

Foram realizadas parcerias com universidades, hospitais, empresas e estabelecimentos comerciais para a realização de palestras com conteúdos gerais de higiene do sono, a influência das tecnologias de comunicação na qualidade do sono e temas específicos do sono em trabalhadores de turno (voltados a esse público em específico). Além disso, também foi realizada parceria com os organizadores da "Caminhada do $2^{\circ}$ Movimento Pro Sono", Bauru/SP.

As ações tiveram o apoio da ABS, tanto com a divulgação das atividades no site oficial e no Facebook, bem como com a disponibilização de cartilhas e bexigas para a distribuição durante as ações.

\section{RESULTADOS}

As diferentes ações de promoção de saúde foram realizadas em cinco cidades do interior do estado de São Paulo, Brasil (Agudos, Bauru, Botucatu, São Manuel e Lençóis Paulista) durante a Semana do Sono do ano de 2017, abrangendo diferentes faixas etárias (crianças, adolescentes, universitários e idosos).

As ações foram realizadas em nove locais, envolvendo funcionários, trabalhadores em turnos; idosos participantes de grupos da melhor idade; universitários, de diferentes cursos de graduação e diferentes universidades; e a população em geral. O Quadro 1 apresenta os detalhes destas ações.

As palestras nas faculdades e na empresa foram realizadas com recurso multimídia, e tiveram duração de 20 a 60 minutos cada. Para as outras ações, foram abordadas as pessoas individualmente, distribuindo cartilhas, explicando o conteúdo, fornecendo algumas dicas e esclarecendo dúvidas. Ao todo, 489 indivíduos foram diretamente abordados e receberam informações sobre a importância do sono de qualidade, além da disponibilização de cartilhas extras nos locais de ações de grande circulação de pessoas.
Quadro 1. Ações de promoção de saúde realizadas durante a Semana do Sono 2017 no interior do estado de São Paulo, Brasil

\begin{tabular}{|c|c|c|c|}
\hline Local & Cidade & $\begin{array}{l}\text { Número de } \\
\text { indivíduos }\end{array}$ & $\begin{array}{l}\text { Perfil dos } \\
\text { indivíduos } \\
\text { abordados }\end{array}$ \\
\hline $\begin{array}{l}\text { Coca-Cola } \\
\text { FEMSA }\end{array}$ & Bauru/SP & 60 & $\begin{array}{c}\text { Trabalhadores } \\
\text { em turnos }\end{array}$ \\
\hline $\begin{array}{ll}\text { Faculdade } & \text { de } \\
\text { Odontologia } & \text { de } \\
\text { Bauru (USP) } & \\
\end{array}$ & Bauru/SP & 38 & Idosos \\
\hline $\begin{array}{l}\text { Universidade do } \\
\text { Sagrado Coração } \\
\text { (USC) }\end{array}$ & Bauru/SP & 31 & $\begin{array}{l}\text { Estudantes } \\
\text { universitários }\end{array}$ \\
\hline "Açaí da Barra" & Bauru/SP & 47 & $\begin{array}{l}\text { População } \\
\text { geral }\end{array}$ \\
\hline $\begin{array}{l}\text { Caminhada do } 2^{\circ} \\
\text { Movimento Pro } \\
\text { Sono }\end{array}$ & Bauru/SP & 50 & $\begin{array}{l}\text { População } \\
\text { geral }\end{array}$ \\
\hline $\begin{array}{l}\text { Faculdade de } \\
\text { Agudos (FAAG) }\end{array}$ & Agudos/SP & 54 & $\begin{array}{c}\text { Estudantes } \\
\text { universitários }\end{array}$ \\
\hline $\begin{array}{l}\text { Faculdade } \\
\text { Marechal Rondon } \\
\text { (FMR) }\end{array}$ & $\begin{array}{c}\text { São } \\
\text { Manuel/SP }\end{array}$ & 31 & $\begin{array}{l}\text { Estudantes } \\
\text { universitários }\end{array}$ \\
\hline $\begin{array}{lr}\text { Hospital } & \text { das } \\
\text { Clínicas } & - \\
\text { Faculdade } & \text { de } \\
\text { Medicina } & \text { de } \\
\text { Botucatu } & \\
\end{array}$ & Botucatu/SP & 92 & $\begin{array}{l}\text { População } \\
\text { geral }\end{array}$ \\
\hline $\begin{array}{l}\text { Hospital Estadual } \\
\text { de Botucatu }\end{array}$ & Botucatu/SP & 52 & $\begin{array}{l}\text { Populaçãa } \\
\text { geral }\end{array}$ \\
\hline Coca-Cola & $\begin{array}{c}\text { Lençóis } \\
\text { Paulista/SP }\end{array}$ & 34 & $\begin{array}{c}\text { Trabalhadores } \\
\text { em turnos }\end{array}$ \\
\hline
\end{tabular}

\section{DISCUSSÃO}

As desordens do sono são reconhecidas atualmente como importantes problemas de saúde pública ${ }^{11}$. De acordo com o National Research Council (2006) ${ }^{12}$, nos EUA, 70 milhões de indivíduos sofrem de pelo menos 1 dos 60 distúrbios do sono reconhecidos. No Brasil, em estudo realizado por Hirotsu et al (2014) $)^{13}$, foi observado que $76 \%$ da população apresenta pelo menos uma queixa de sono, mostrando que aproximadamente 108 milhões de brasileiros são afetados por distúrbios do sono.

Os estudos demonstram, porém, baixas taxas de diagnóstico das alterações do sono. Estima-se, por exemplo, que a apneia obstrutiva do sono (AOS), um distúrbio de alta prevalência na população e que apresenta sérias consequências, é diagnosticada em apenas $5 \%$ dos indivíduos acometidos. Os $95 \%$ restantes não são diagnosticados por falta de conhecimento da população geral, ou por falta de conhecimento sobre distúrbios do sono por parte dos próprios profissionais da saúde ${ }^{14}$.

Neste contexto, considerando a alta prevalência de alterações do sono e as baixas taxas de diagnóstico, são necessárias ações de promoção de saúde sobre o tema, de modo a conscientizar a população sobre a importância do sono de qualidade e a importância de se procurar um profissional especializado na presença de sinais e sintomas de distúrbios. A "Semana do Sono", realizada anualmente no mundo todo, tem o objetivo de realizar estas ações de promoção de saúde ${ }^{10}$.

No Brasil, a Semana do Sono de 2017, contou com a participação de pelo menos 19 cidades do país ${ }^{10}$. Os resultados do presente estudo mostram que, só no interior do Estado de São Paulo, pelo menos 489 indivíduos, de diferentes faixas etárias, receberam informações sobre a importância do sono de qualidade e sobre os principais sinais e sintomas de distúrbios do sono. Ações como esta são importantes para propagação de informações sobre o tema e esclarecimentos da população ${ }^{9}$. Nós antecipamos que, após as atividades da Semana do Sono 2017, os autores do presente estudo foram convidados a proferir palestras sobre o tema para outros grupos de funcionários que não foram abordados 
durante a semana do sono, principalmente trabalhadores em turnos, em risco para desordens do sono. Isto demonstra que as ações realizadas agregaram conhecimentos à população abordada, provocando a conscientização sobre os distúrbios do sono.

É importante ressaltar que as ações abrangeram diferentes faixas etárias e diferentes perfis de indivíduos, que apresentam particularidades quanto ao risco para distúrbios do sono, com diferentes consequências ${ }^{9}$. Foram abordados, por exemplo, trabalhadores em turno, que frequentemente apresentam distúrbios relacionados ao ciclo circadiano sonovigília e, estes, por sua vez, podem estar associados a acidentes de trabalho ${ }^{15}$. Foram também abordados estudantes universitários, população na qual o sono de má qualidade pode prejudicar o desempenho acadêmico ${ }^{16}$. Foram, ainda, abordados idosos, entre os quais mais da metade da população apresenta distúrbios do sono $^{17}$, principalmente apneia obstrutiva do sono.

$\mathrm{Na}$ literatura, encontra-se a intervenção realizada em professores do ensino básico, que também gerou resultados expressivos quanto à modificação de hábitos de sono ${ }^{18}$, bem como a intervenção com alunos do ensino médio ${ }^{19}$. Desta forma, sugere-se que novas ações de promoção de saúde nesta temática sejam realizadas com número ainda maior de indivíduos abordados, e abrangendo diferentes perfis populacionais.

\section{CONCLUSÃO}

Nota-se a importância da semana do sono para a propagação de informações sobre o sono e esclarecimentos da população. As ações do interior do estado de São Paulo ocorreram de modo abrangente, atuando na população universitária até com grupos de idosos. Sugere-se que ações como esta sejam repetidas.

\section{AGRADECIMENTOS}

Ao apoio e parceria da Associação Brasileira do Sono.

\section{REFERÊNCIAS}

1. American Academy of Sleep Medicine. The AASM manual for the scoring of sleep and associated events: rules, terminology and technical specifications. $1^{\text {a }}$ ed.: Westchester, Illinois. 2014. 59 p.

2. Tufik S, Santos-Silva R, Taddei JA, Bittencourt LR. Obstructive sleep apnea syndrome in the Sao Paulo epidemiologic sleep study. Sleep Med. 2010; 11(5):441-6.

3. Krieger J, McNicholas WT, Levy P, De Backer W, Douglas N, Marrone $\mathrm{O}$ et al. Public health and medicolegal implications of sleep apnoea. Eur Respir J. 2002; 20(6):1594-609.

4. Akkoyunlu ME, Kart L, Uludag M, Bayram M, Alisha G, Özçelik $\mathrm{H}$ et al. Relationship between symptoms of obstructive sleep apnea syndrome and traffic accidents in the city drivers. Tuberk Toraks. 2013; 61(1):33-7.

5. Rajaratnam SM, Barger LK, Lockley SW, Shea SA, Wang W, Landrigan CP et al. Sleep disorders, health, and safety in police officers. JAMA. 2011; 306(23):2567-78.

6. Drager LF, Genta PR, Pedrosa RP, Nerbass FB, Gonzaga $\mathrm{CC}$, Krieger EM et al. Characteristics and predictors of obstructive sleep apnea in patients with systemic hypertension. Am J Cardiol. 2010; 105(8):1135-9.

7. Punjabi N, Caffo B, Goodwin J, Gottlieb D, Newman A, O'Connor $G$ et al. Sleep- disordered breathing and mortality: A prospective cohort study. PLoS Med. 2009; 6(8):e1000132.

8. Smith SS, Lang CP, Sullivan KA, Warren J. A preliminary investigation of the effectiveness of a sleep apnea education program. J Psychosom Res. 2004; 56(2):245-9.
9. Corrêa CC, Blasca WQ, Berretin-Felix G. Health promotion in obstructive sleep apnea syndrome. Int Arch Otorhinolaryngol. 2015; 19(2):166-70.

10. ABS - Associação Brasileira de Sono [homepage na internet]. São Paulo: Associação Brasileira de Sono [acesso em 2018 jan 08]. Disponível em: http://www.absono.com.br

11. Filomeno R, Ikeda A, Tanigawa T. Developing policy regarding obstructive sleep apnea and driving among commercial drivers in the United States and Japan. Ind Health. 2016; 54(5):469-75.

12. National Research Council. Sleep disorders and sleep deprivation: an unmet public health problem. Washington, DC: National Academies Press, 2006.

13. Hirotsu C, Bittencourt L, Garbuio S, Andersen ML, Tufik S. Sleep complaints in the Brazilian population: Impact of socioeconomic factors. Sleep Sci. 2014; 7(3):135-42.

14. Fuhrman C, Fleury B, Nguyên XL, Delmas MC. Symptoms of sleep apnea syndrome: high prevalence and underdiagnosis in the French population. Sleep Med 2012; 13(7):852-8.

15. Rodenstein D. Sleep apnea: traffic and occupational accidents--individual risks, socioeconomic and legal implications. Respiration. 2009; 78(3):241-8.

16. Enright T, Refinetti R. Chronotype, class times, and academic achievement of university students. Chronobiol Int. 2017; 34(4):445-50.

17. Foley DJ. Sleep Complaints among elderly persons: an epidemiologic study of three communities. Sleep, 1995; 18(6):425-32.

18. Souza JC, Galina SD, Sousa IC, Azevedo CVM. Effect of a sleep education program on sleep knowledge and habits in elementary and high school teachers. Estud Psicol (Natal). 2016; 21(4):369-80.

19. Corrêa CC, Berretin-Felix G, Blasca WQ. Educational program applied to obstructive sleep apnea. Journal of Communication Disorders, Deaf Studies \& Hearing Aids. 2016; 4:1-6

\section{CONFLITO DE INTERESSES}

Os autores declaram não haver conflitos de interesse.

\section{AUTOR PARA CORRESPONDÊNCIA}

\section{Camila de Castro Corrêa}

camila.ccorrea@hotmail.com

Submetido em 08/01/2018 Aceito em 10/02/2018 